\title{
Needle-Type Glucose Sensor Based on Functionalized Graphene
}

\author{
K. ul Hasan*, M. H Asif*, O Nur and M Willande
}

Department of Science and Technology, Campus Norrköping, Linköping University, SE-60174 Norrköping Sweden

\begin{abstract}
We demonstrate a novel, highly efficient glucose sensor based on functionalized graphene. Glucose oxidase (GOD) immobilization has been apprehendedbythe direct interaction between carboxyl acid groups of the reduced graphene oxide (RGO) and amines of GOD together with the electrostatic interactions existing between the positively charged polymeric ionic liquid (PIL) and GOD. This combined system can provide a favorable microenvironment for the GOD to retain its good bioactivity. The enzyme-coated graphene biosensor exhibited glucose-dependent electrochemical measurements against an $\mathrm{Ag} / \mathrm{AgCl}$ reference electrode. The resulting sensor show broad range detection, up to $100 \mathrm{mM}$ glucose concentration, with a sensitivity of $5.59 \mu \mathrm{A} /$ decade. It was found that glucose biosensor based on functionalized graphene can be seen as an effective candidate for the detection of sugar concentration in clinical diagnoses.
\end{abstract}

\section{Introduction}

Graphene has drawn significant attention since its discovery in 2004, not only in the field of basic research but also in technological applications due to its unique physicochemical dimensions, high sensitivity and excellent mechanical, thermal and electrical properties [1-5]. Development of the biosensors can potentially be an interesting application of graphene in order to utilize its tremendously large surface area to volume ratio as a dominating and promising parameter $[6,7]$.There are many analytical procedures for the detection of biomolecules but enzymatic electrochemical biosensing technique is being hailed as an alternative to the non-enzymatic biosensing. Preparation of enzymatic biosensors could be achieved through the immobilization of various types of enzymes onto the biocompatible matrices depending on the target molecules. The immobilization of enzymes onto the surfaces of suitably modified electrodes can significantly improve the bioactivity due to the biocompatibility of the surface modifier materials. The dramatic decrease in the hydrogen peroxide over potential and the direct electron transfer of glucose oxidase (GOD) observed at carbon nanotube (CNT) modified electrodes have already shown great promise for the biosensing of glucose $[8,9]$. Graphene based chemical sensors can potentially have a much higher sensitivity because graphene is a 2-dimentional single atomic layer of graphite which can maximize the interaction between the surface dopants and adsorbates. It has much lower Johnson noise [10-12] as compared to CNT, therefore, a minute variation of carrier concentration can cause a notable variation of electrical conductivity [13]. Moreover, graphene can be obtained easily by chemical conversion of inexpensive graphite [14].

GOD is widely employed in most of the glucose biosensors due to its high selectivity to glucose. The various methods such as covalent bonding [15], embedding method [16] and cross linking method [1719] have been used to immobilize the GOD onto different supporting materials.

One popular route of graphene synthesis is through graphite oxide [20] which can be exfoliated to graphene oxide (GO) [21]. GO contains large amounts of oxygen groups, which can be favorable to the functionalization through the action of the biomolecules for biorecognition events during biosensing [22,23]. Graphene oxide can be reduced chemically or electrochemically and this reduced graphene oxide (RGO) has a partly restored $\mathrm{sp}^{2}$ lattice but still holds some fraction of oxygen-containing groups.

The GO aqueous dispersion was reduced with hydrazine in the presence of a polymeric ionic liquid (PIL) [24]. PILs are low molecular weight imidazolium salts with a low melting point. We use dpolyquaternium 16 (BASF Ludwigshafen, Germany) which is a copolymer with $95 \%$ molar of imidazolium chloride and 5\% molar of vinylimidazole. Ionic liquids (ILs) have many advantageous properties such as non-flammability, a wide electrochemical window, high thermal stability, wide liquid range, and small vapor pressure [25]. PILs are also known to interact strongly with the basal plane of graphite and graphene, resulting in a stable aqueous dispersion.

We prepare the biosensor working electrode on a metal wire contrary to the conventional glassy carbon flat substrates reported previously for grapheme $[14,23,26-28]$. Main motive behind this is to take the biosensing to the intracellular environment with this needle like graphene biosensor. Glucose biosensors based on PIL functionalized graphene have been reported recently [28] by using common glassy carbon substrates. We utilize commercial polyquaterniums which are not reported previously in graphene functionalization and biosensing. These polyquaterniumsare specifically used in conditioners, shampoos, hair mousse, hair sprays, hair dye and contact lens solutions because of being positively charged [29]. They neutralize the negative charges of most shampoos and hair proteins and help the hair lie flat. This property can be extremely advantageous for enhancing GOD immobilization a step further as GOD has a negative charge in neutral $\mathrm{pH}$ solutions. This positively charged ionic liquid is well capable of the electrostatic incorporation of a negatively charged glucose oxidase enzyme and results in an efficient glucose sensing device [30]. GOD

${ }^{*}$ Corresponding authors: Kamran ul Hasan, Department of Science and Technology, Campus Norrköping, Linköping University, SE-60174 Norrköping Sweden, Tel: +4611363472; Fax: +4611363270; E-mail: kamran.ul.hasan@liu.se

M.H. Asif, Department of Science and Technology, Campus Norrköping, Linköping University, SE-60174 Norrköping Sweden, Tel: +4611363472; Fax: +4611363270; E-mail: muhas@itn.liu.se

Received October 26, 2011; Accepted December 20, 2011; Published December 28, 2011

Citation: ul Hasan K, Asif MH, Nur O, Willander M (2012) Needle-Type Glucose Sensor Based on Functionalized Graphene. J Biosens Bioelectron 3:114 doi:10.4172/2155-6210.1000114

Copyright: @ 2012 ul Hasan K, et al. This is an open-access article distributed under the terms of the Creative Commons Attribution License, which permits unrestricted use, distribution, and reproduction in any medium, provided the original author and source are credited. 
is also immobilized into the GO sheets via peptide bonds between the amine groups of GOD and the residual carboxylic functionalities of GO [27].

Therefore, by combining these two mechanisms of immobilization (Schematic Figure $1 \mathrm{ab}$ ), we can have a big advantage in using the functionalized graphene for the right application (as enzymatic biosensors) and transduction mechanism.

\section{Materials and Methods}

Graphene oxide was prepared by the modified Hummer's method [20,21]. The graphite flakes (Sigma Aldrich PN 332461, 4 g) were first put in $\mathrm{H}_{2} \mathrm{SO}_{4}(98 \%, 12 \mathrm{~mL})$ and kept at $80^{\circ} \mathrm{C}$ for $5 \mathrm{~h}$. The resulting solution was cooled down to room temperature. Mild sonication was performed in a water bath for $2 \mathrm{~h}$ to further delaminate graphite into a few micron flakes. Sonication time and power is very critical as it defines the size of the resulting graphene oxide sheets. Excessive sonication leads to extremely small flakes. Then the solution was diluted with $0.5 \mathrm{~L}$ deionized (DI) water and left overnight. The solution was filtered by Nylon Millipore filters afterwards. The resulting powder was mixed with $\mathrm{KMnO}_{4}$ and $\mathrm{H}_{2} \mathrm{SO}_{4}$ and put in a cooling bath under constant stirring for $1.5 \mathrm{~h}$. The solution was diluted with DI water and $20 \mathrm{~mL} \mathrm{H}_{2} \mathrm{O}_{2}(30 \%)$ was added to it.

The supernatant was collected after $12 \mathrm{~h}$ and dispersed in dilute $\mathrm{HCl}$ in order to remove the metal ion residue and then recovering by centrifuge $[20,21]$. Recovered clean GO was again dispersed in water to make a homogeneous dispersion and was centrifuged at $8000 \mathrm{rpm}$ for 40 mins in order to remove the multilayer fragments. A PIL was added into the aqueous dispersion of GO at a concentration of $1 \mathrm{mg}$ $\mathrm{mL}^{-1}$, followed by reduction by hydrazine [24]. This process resulted in aqueous dispersion of functionalized graphene sheets.

Graphene was coated on a hot platinum (Pt) wire by dip coating [31,32]. Glucose oxidase (GOD)enzymesolution, $10 \mathrm{mg} / \mathrm{ml}$, was prepared in $10 \mathrm{mM}$ Phosphate Buffered Saline, pH 7.4 using GOD (E.C. 1.1.3.4) from Aspergillus niger type GO3A, $360 \mathrm{U} / \mathrm{mg}$ (BBI Enzymes Ltd). GOD enzyme was electrostatically immobilized by dipping the graphene-coated metal wire into $10 \mathrm{~mL}$ of the enzyme solution for 15 minutes at room temperature and then it was dried in air for approximately 20 minutes. All the electrochemical measurements of GOD immobilized graphene were perform usinga Keithley 2400 source meter, connected via computer controlled programin glucose solutions ranging from $1 \mu \mathrm{M}-100 \mathrm{mMversus}$ an $\mathrm{Ag} / \mathrm{AgCl}$ as reference electrode.

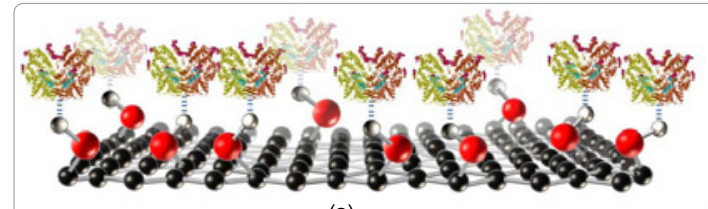

(a)
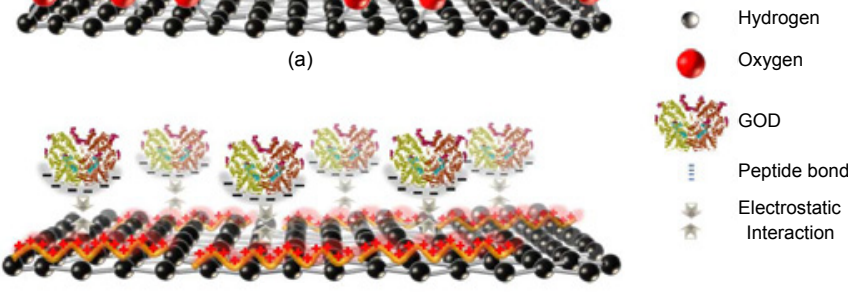

Figure 1: Schematic of the GOD immobilization on graphene(a) Peptide bond formation between remaining carboxylic groups of RGO and GOD (b)Electrostatic interaction between PIL functionalized graphene surface and GOD.

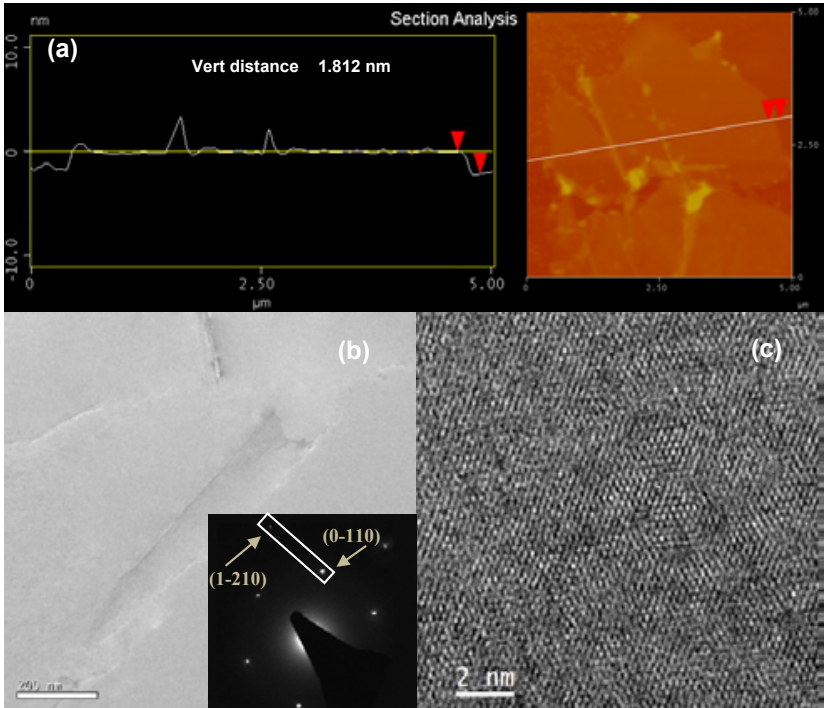

Figure 2: (a) AFM image of graphene nanosheets showing height profile(b) Low resolution TEM image of a typical wrinkled graphene sheet. Inset shows hexagonal skeleton carbon ring arrangement of carbon atom. (c) High resolution TEM illustrates good crystal quality of nanosheet.

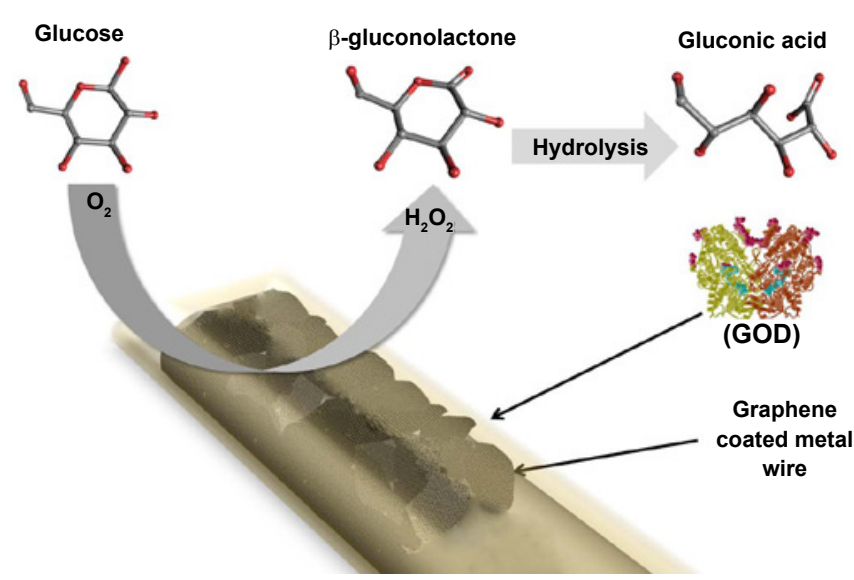

Figure 3: Schematic illustration of the glucose sensing setup using working electrode comprised of graphene sheets coated with GOD, along with possible electrochemical reaction near the electrode.

\section{Results and Discussion}

AFM image of our chemically reduced GO is shown in Figure 2. The graphene sheets were found to be fractionally thicker than the theoretical thickness of a single graphene layer owing to the adsorption of the PIL on the surface of the graphene sheets by non-covalent $\pi-\pi$ interactions between the imidazolium rings of the PIL and graphene sheets $[24,33,34]$. The physical adsorption the ionic liquids along the surface of the graphene sheets has been verified by X-ray photoelectron spectroscopy (XPS) analysis, previously [34].

The sample for the transmission electron microscopy (TEM) was prepared by simple drop casting of the graphene dispersion onto the holey carbon grid. A fan heater was utilized with an objective to dry the holey carbon grid containing graphene nanosheets. The bright-field low resolution TEM results are shown in figure 2(b); selected area electron diffraction (SAED) pattern displays good crystalline quality of graphene 
nanosheets with regular arrangement of hexagonal atomic packing of carbon, inset of Figure 2(b). The method of diffraction intensity ratios in the SAED pattern agrees with the previously reported results related to mono-layer graphene nanosheets [35]. High resolution TEM image demonstrates the presence of carbon lattice in Figure 2(c).

Graphene deposition onto the Pt wire was carried out by dipcoating. The metal wire can be dipped into the graphene solution for some time so as to allow the absorption of graphene planes on the surface of the metal [31,32]. The binding force between graphene and $\mathrm{Pt}, \mathrm{Pd}$ and $\mathrm{Ni}$ surface is found to be higher than that for other metal surfaces [36]. Recent experimental observations also suggest a relatively strong adhesion between the metal (Pt and Pd) and RGO [37].

Selective glucose measurements were performed by atwo electrode electrochemical method. A Graphene-decorated electrode coated with enzyme served as the working electrode, and an $\mathrm{Ag} / \mathrm{AgCl}$ electrode was used as reference electrode. The electrochemical measurements were started 30 minutes after immobilizing the graphene working electrode with GOD. The response of the glucose probe was measured with a Keithley 2400 source meter, connected via computer controlled lab view program. The construction of a two-electrode electrochemical cell was as follows:

reference electrode | reference electrolyte || test electrolyte | indicator electrode

Amperometry is relatively sensitive electrochemical technique; the output signal is the current that is linearly dependent on the analyte concentration by applying a constant biased. Graphene decorated electrode was tested amperometrically to measure the current at an accumulation voltage of $1 \mathrm{~V}$. Good sensitivity and selectivity has been realized by proper binding of GOD enzyme with functionalized graphene sheets. The analyte solution modification corresponds to abrupt current change and these changes can be related to the concentration of ions in the test electrolyte via a calibration procedure. Figure 4a depicts the calibration curve of the current versus concentration of the glucose ranging from $1 \mu \mathrm{M}$ to $100 \mathrm{mM}$. We see that the sensor deviates from the linearity for the concentrations above $1000 \mu \mathrm{M}$ but shows very good linear behavior below that limit. A good linearity up to $1000 \mu \mathrm{M}$ very easily covers the clinical range of glucose in human blood [38]. Inset of the Figure 4 s displays the results of three experiments for the same glucose-sensing electrode for the range between $15 \mu \mathrm{M}$ to $1000 \mu \mathrm{M}$. It shows good repeatability and linearity for various glucose concentrations in this range. The sensitivity of the enzyme immobilized graphene electrode is determined by the slope of the calibration curve and was found to be $5.59 \mu \mathrm{A} /$ decade. A constant potential is applied to the GOD immobilized graphene electrode which assists hydrogen peroxide production from the oxidation of glucose. To test the sensitivity and stability of our constructed graphene based glucose sensor, we performed measurements while changing the glucose concentration in the buffer solution around the working electrode. Current response at the successive addition of glucose is recorded as shown in Figure $4 \mathrm{~b}$. The current at the graphene modified electrode increased with the gradual injection of glucose, pointing out that the current response arises from the oxidation of hydrogen peroxide produced during the enzyme reaction rather than from direct oxidation of glucose at the electrode [27]. The output response with stability is shown in Figure 5, indicating that the proposed sensor is fast and sufficiently stable.

After the amperometric measurements, the GOD immobilized GO electrode was tested potentiometrically. The response of the electrochemical potential difference of the GOD-graphene/metal to the changes in buffer electrolyte glucose was measured for the range of $1 \mu \mathrm{M}$ to $100 \mathrm{mM}$ and shows that this glucose dependence is linear and has sensitivity equal to $1.18 \mathrm{~V} /$ decade at $23^{\circ} \mathrm{C}$ (Figure 6). This linear dependence implies that the sensor configuration can provide a large dynamic range.

The sensing mechanism of the electrochemical glucose sensors is based on an enzymatic reaction catalysed by glucose oxidase (GOD) with $\beta \mathrm{d}$-glucose, according to the following reaction:

$$
\mathrm{H}_{2} \mathrm{O}+\mathrm{O}_{2}+\mathrm{d} \text {-Glucose } \stackrel{\text { GOD }}{\longrightarrow} \mathrm{d} \text {-gluconolactone }+\mathrm{H}_{2} \mathrm{O}_{2}(1)
$$

As a result of this reaction, $\mathrm{d}$-gluconolactone and hydrogen peroxide are produced. These two products with the oxygen consumption can be used for glucose determination. With $\mathrm{H}_{2} \mathrm{O}$ availability in the reaction, gluconolactone is spontaneously converted to gluconic acid (by hydrolysis reaction), resulting in a decrease of $\mathrm{pH}$ was used for the indirect determination of the glucose concentration with charged
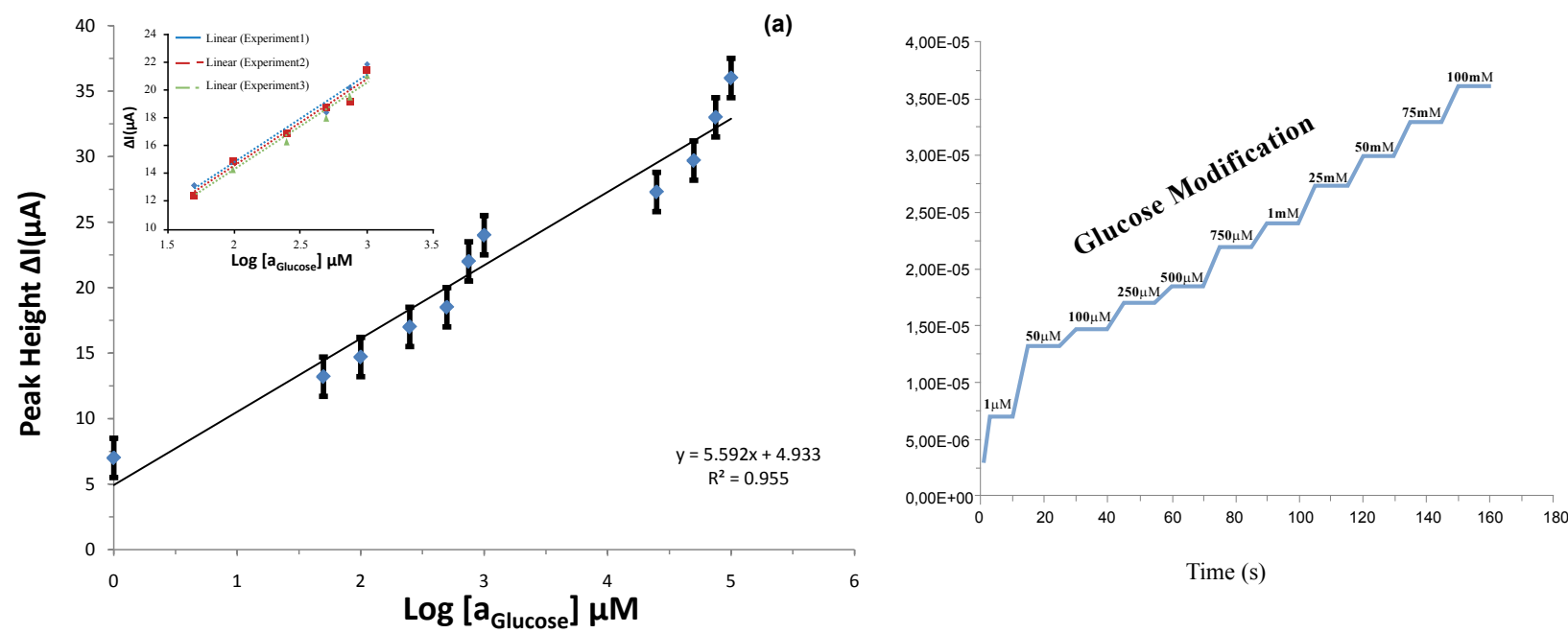

Figure 4: (a) Typical amperometric response at the GOD immobilized graphene decorated electrode versus glucose concentration, (b)The amperometric response versus time as the glucose concentration is modified surrounding the graphene decorated electrode. 
Citation: ul Hasan K, Asif MH, Nur O, Willander M (2012) Needle-Type Glucose Sensor Based on Functionalized Graphene. J Biosens Bioelectron 3:114. doi:10.4172/2155-6210.1000114

Page 4 of 5

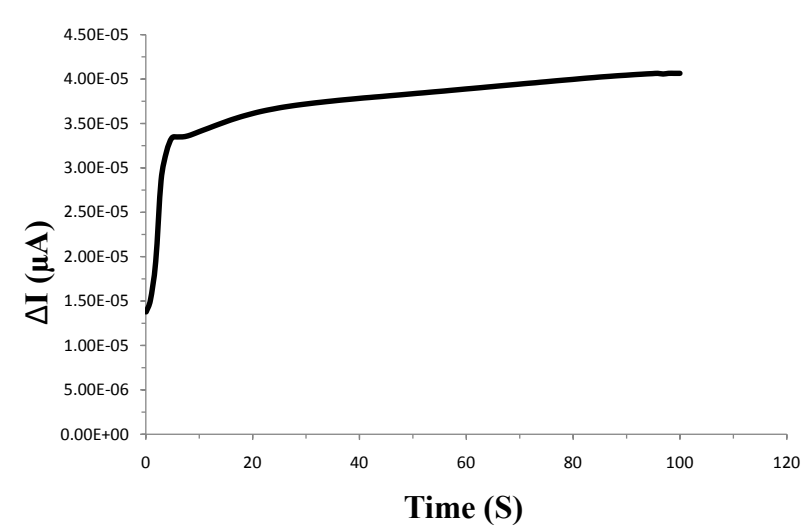

Figure 5: The output response versus time showing the stability of the sensor.

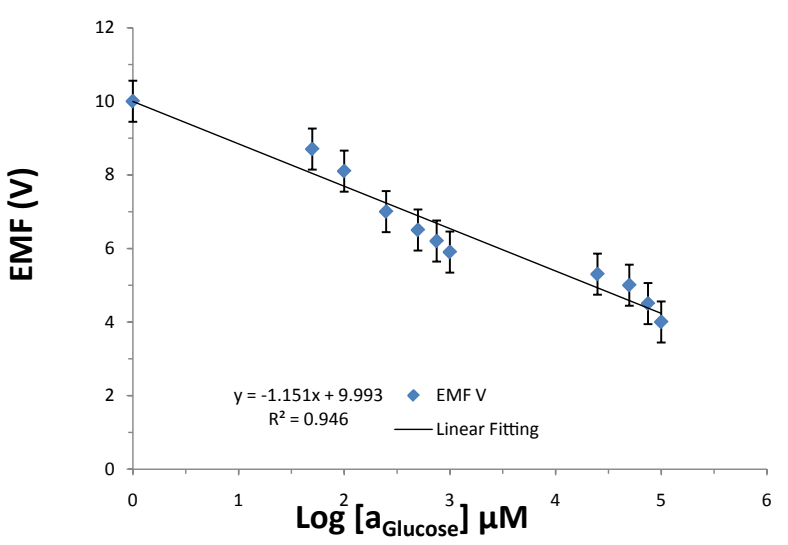

Figure 6: Calibration curve showing the electrochemical potential difference for the enzyme coated graphene electrode with $\mathrm{Ag} / \mathrm{AgCl}$ reference electrode versus Logarithmic concentration range for glucose solution variation over the range of $1 \mu \mathrm{M}$ to $100 \mathrm{mM}$.

products of gluconate ${ }^{-}$and a proton $\left(\mathrm{H}^{+}\right)$according to the following equation:

$\mathrm{d}$ - Gluconolactone Gluconate ${ }^{-}+\mathrm{H}^{+} \stackrel{\text { Spontaneous }}{\longrightarrow}(2)$

Typical biosensors usually measure the amount of hydrogen peroxide produced by the reaction between glucose and GO. Alternatively, some sensors (including our biosensor) measure the amount of oxygen consumed, or a change in $\mathrm{pH}$ which is accompanied by the production of gluconic acid that is produced from gluconolactone show in equation (2) [38-40].

\section{Conclusion}

In summary, we demonstrate a novel and efficient glucose sensor based on functionalized graphene. Interaction between carboxyl acid groups of RGO and amines of glucose oxidase plus the presence of positively charged PIL resulted in an effective GOD immobilization. The resulting electrochemical sensor exhibits a broad linear range up to $100 \mathrm{mM}$ glucose concentration and with a sensitivity of $5.59 \mu \mathrm{A} /$ decade and a stable output response. Thus, sensor based on functionalized graphene can be seen as an effective candidate for the detection of sugar concentration, paving the way to its potential applications in the clinical diagnosis.

\section{References}

1. Balandin AA, Ghosh S, Bao W, Calizo I, Teweldebrhan D, et al. (2008) Superior thermal conductivity of single-layer graphene. Nano Letters 8: 902-907.

2. Chen H, Muller MB, Gilmore KJ, Wallace GG, Li D (2008) Mechanically strong, electrically conductive, and biocompatible graphene paper. Adv Mater 20: 3557-3561.

3. Du X, Skachko I, Barker A, Andrei EY (2008) Approaching ballistic transport in suspended graphene. Nat Nanotech 3: 491-495.

4. Freitag M, Chiu HY, Steiner M, Perebeinos V, Avouris P (2010) Therma infrared emission from biased graphene. Nat Nanotech 5: 497-501.

5. Garaj S, Hubbard W, Reina A, Kong J, Branton D, et al. (2010) Graphene as a subnanometre trans-electrode membrane. Nature 467: 190-193.

6. Park S, Ruoff RS (2009) Chemical methods for the production of graphenes Nat Nanotech 4: 217-224.

7. Stoller MD, Park SJ, Zhu YW, An JH, Ruoff RS (2008) Graphene-based ultracapacitors. Nano Lett 8: 3498-3502.

8. Xu L, Zhu Y, Tang L, Yang X, Li C (2007) Biosensor based on self-assembling glucose oxidase and dendrimer-encapsulated pt nanoparticles on carbon nanotubes for glucose detection. Electroanalysis 19: 717-722.

9. Wang J (2005) Carbon-nanotube based electrochemical biosensors: a review. Electroanalysis 17: 7-14

10. Geim AK, Novoselov KS (2007) The rise of graphene. Nat Mater 6: 183-191.

11. Novoselov KS, Geim AK, Morozov SV, Jiang D, Katsnelson MI, et al. (2005) Two-dimensional gas of massless dirac fermions in graphene. Nature 438 197-200.

12. Zhang YH, Ruan WJ, Li ZY, Wu Y, Zheng JY (2005) DFT study on the influence of meso-phenyl substitution on the geometric, electronic structure and vibrational spectra of free base porphyrin. Chemical Physics 315: 201-213.

13. Ao ZM, Yang J, Li S, Jiang Q (2008) Enhancement of CO detection in Al doped graphene. Chem Phys Lett 461: 276-279.

14. Xu C, Wang X, Zhu J (2008) Graphene - Metal particle nanocomposites. J Phys Chem C 112: 19841-19845.

15. Piro B, Do VA, Le LA, Hedayatullah M, Pham MC (2000) Electrosynthesis of a new enzyme-modified electrode for the amperometric detection of glucose. $J$ Electroanal Chem 486: 133-140.

16. Cosnier S, Senillou A, Grätzel M, Comte P, Vlachopoulos N, et al. (1999) A glucose biosensor based on enzyme entrapment within polypyrrole films electrodeposited on mesoporous titanium dioxide. J Electroanal Chem 469 $176-181$.

17. Muguruma H, Hiratsuka A, Karube I (2000) Thin-film glucose biosensor based on plasma-polymerized film: simple design for mass production. Anal Chem 72: $2671-2675$.

18. Yang Q, Atanasov P, Wilkins E (1998) Development of needle-type glucose sensor with high selectivity. Sens Actuators B: Chemical 46: 249-256.

19. Wu B, Zhang G, Shuang S, Choi MMF (2004) Biosensors for determination of glucose with glucose oxidase immobilized on an egg shell membrane. Talanta 64: $546-553$

20. Hummers WS, Offeman RE (1958) Preparation of graphitic oxide. Journal of the American Chemical Society 80: 1339-1339.

21. Dong X, Su CY, Zhang W, Zhao J, Ling Q, et al. (2010) Ultra-large singlelayer graphene obtained from solution chemical reduction and its electrical properties. Phys Chem Chem Phys 12: 2164-2169.

22. Dreyer DR, Ruoff RS, Bielawski CW (2010) From conception to realization: an historial account of graphene and some perspectives for its future. Angewandte Chemie International Edition 49: 9336-9344.

23. Pumera M (2011) Graphene in biosensing. Materials Today 14: 308-315.

24. ul Hasan K, Sandberg M, Nur O, Willander M (2011) Polycation stabilization of graphene suspensions. Nanoscale Res Lett 6: 493.

25. Carrión F, Sanes J, Bermúdez MD, Arribas A (2010) New single-walled carbon nanotubes-ionic liquid lubricant. Application to polycarbonate-stainless steel sliding contact. Tribol Lett 41: 199-207. 
Citation: ul Hasan K, Asif MH, Nur O, Willander M (2012) Needle-Type Glucose Sensor Based on Functionalized Graphene. J Biosens Bioelectron 3:114. doi:10.4172/2155-6210.1000114

Page 5 of 5

26. Gan T, Hu S (2011) Electrochemical sensors based on graphene materials Microchimica Acta 175: 1-19.

27. Liu Y, Yu D, Zeng C, Miao Z, Dai L (2010) Biocompatible graphene oxidebased glucose biosensors. Langmuir 26: 6158-6160.

28. Zhang Q, Wu S, Zhang L, Lu J, Verproot F, et al. (2011) Fabrication of polymeric ionic liquid/graphene nanocomposite for glucose oxidase immobilization and direct electrochemistry. Biosens Bioelectron 26: 2632-2637.

29. Andre Barel MP, Howard I, Maibach (2009) Handbook of Cosmetic Science and Technology, 869 (Informa Healthcare USA, Inc, New York, NY 10017)

30. Pumera M (2009) Electrochemistry of graphene: new horizons for sensing and energy storage. Chem Rec 9: 211-223.

31. Penicaud A \& Valles C. GRAPHENE SOLUTIONS. (Centre National De La Recherche Scientifique - CNRS, Paris Cedex I6 (FR), US, 2011).

32. Zhou H, Wang X, Yu P, Chen X, Mao L (2011) Sensitive and selective voltammetric measurement of $\mathrm{Hg}^{2+}$ by rational covalent functionalization of graphene oxide with cysteamine. Analyst 137: 305-308

33. Kim T, Lee H, Kim J, Suh KS (2010) Synthesis of phase transferable graphene sheets using ionic liquid polymers. ACS Nano 4: 1612-1618.
34. Zhou X, Wu T, Ding K, Hu B, Hou M, et al. (2009) Dispersion of graphene sheets in ionic liquid [bmim][PF6] stabilized by an ionic liquid polymer. Chemical Communications 46: 386-388.

35. Meyer JC, Geimc AK, Katsnelsond MI, Novoselovc KS, Obergfelle D, et al. (2007) On the roughness of single- and bi-layer graphene membranes. Solid State Communications 143: 101-109.

36. Wintterlin J, Bocquet ML (2009) Graphene on metal surfaces. Surface Science 603: $1841-1852$.

37. Pandey PA, Bell GR, Rourke JP, Sanchez AM, Elkin MD, et al. (2011) Physical vapor deposition of metal nanoparticles on chemically modified graphene: observations on metal-graphene interactions. Small 7: 3202-3210.

38. Macaya DJ, Nikolou M, Takamatsu S, Mabeck JT, Owens RM, et al. (2007) Simple glucose sensors with micromolar sensitivity based on organic electrochemical transistors. Sens Actuators B Chem 123: 374-378.

39. Cunningham AJ (1998) Introduction to Bioanalytical Sensors 318 (Wiley, New York)

40. Hanazato Y, Inatomi KI, Nakako M, Shiono S, Maeda M (1988) Glucosesensitive field-effect transistor with a membrane containing co-immobilized gluconolactonase and glucose oxidase. Analytica Chimica Acta 212: 49-59. 